\title{
Tuning the surface microstructure of titanate coatings on titanium implants for enhancing bioactivity of implants
}

This article was published in the following Dove Press journal:

International Journal of Nanomedicine

8 June 2015

Number of times this article has been viewed

\author{
Hui Wang ${ }^{1,2}$ \\ Yue-Kun Lai' \\ Ru-Yue Zheng' \\ Ye Bian' \\ Ke-Qin Zhang' \\ Chang-Jian Lin ${ }^{2}$
}

'National Engineering Laboratory for Modern Silk, College of Textile and Clothing Engineering, Soochow University, Suzhou, ${ }^{2}$ State Key Laboratory for Physical Chemistry of Solid Surfaces, Department of Chemistry, College of Chemistry and Chemical Engineering, Xiamen University, Xiamen, People's Republic of China
Correspondence: Hui Wang National Engineering Laboratory for Modern Silk, College of Textile and Clothing Engineering, Soochow University, Suzhou 215123,

People's Republic of China

Tel +865126706 II69

Fax +8651267061129

Email whui@suda.edu.cn
Abstract: Biological performance of artificial implant materials is closely related to their surface characteristics, such as microtopography, and composition. Therefore, convenient fabrication of artificial implant materials with a cell-friendly surface structure and suitable composition was of great significance for current tissue engineering. In this work, titanate materials with a nanotubular structure were successfully fabricated through a simple chemical treatment. Immersion test in a simulated body fluid and in vitro cell culture were used to evaluate the biological performance of the treated samples. The results demonstrate that the titanate layer with a nanotubular structure on Ti substrates can promote the apatite-inducing ability remarkably and greatly enhance cellular responses. This highlights the potential of such titanate biomaterials with the special nanoscale structure and effective surface composition for biomedical applications such as bone implants.

Keywords: nanotubes structure, bone-like apatite, cell culture, biological performance

\section{Introduction}

In biomaterials science, the fabrication of bioscaffolds with both excellent biological performances and strong mechanical properties is a longstanding challenge. Especially, when implanted into hard tissue, like bone, the biomaterial should be strong enough to support considerable weight. Titanium and its alloys have been widely used for synthetic metallic implants in hard tissues due to their low toxicity, good biocompatibility, favorable mechanical properties, and great resistance to the corrosion compared to other metals. ${ }^{1-4}$ However, although untreated implantable titanium with a smooth surface can support weight, it may lack the interspaces to accommodate tissue growth and may be unable to firmly bond with the bone and promote new bone formation on the surface, which may lead to the slow fixation of the implant and its gradual loosening over time. Hopefully, the surface modification of titanium and its alloys can optimize the biological performance of Ti-based biomaterials, and at the same time retain their favorable mechanical properties. ${ }^{4-10}$ When implanted into a living body, the surface physicochemical properties of the biomaterials determine the interactions between them and the surrounding biological environment. ${ }^{10-12}$ Recent efforts in this field have highlighted the importance of modifying the surface topography and composition of materials to achieve good biological performance of implants. ${ }^{13-15}$ In the past decade, design and fabrication of nanostructured titania and titanate layers on titanium have attracted much attention because of the improved biological performance of titanium and its widespread application in bone implants. ${ }^{14-18}$ Generally, 
conventional titania and titanate nanostructures can be achieved by the sol-gel, ${ }^{19,20}$ sputtering, ${ }^{21,22}$ electrophoretic, ${ }^{23,24}$ chemical vapor deposition, ${ }^{25}$ anodization, ${ }^{26,27}$ or hydrothermal methods. ${ }^{28-31}$ Among these techniques, hydrothermal method is one of the most facile techniques due to its simplicity, cost-effectiveness, and suitability for mass production. Wu and coworkers reported obtaining $\mathrm{H}_{2} \mathrm{Ti}_{3} \mathrm{O}_{7}$ powders with nanotubes, nanofibers, nanowires, and nanobelt structures by the alkali hydrothermal method. ${ }^{29}$ Wang also reported that titanate nanobelt powders with tunable wettability provide the possibility for chemical sensors, which were prepared using a modified hydrothermal method. ${ }^{27}$ The nanostructure of titania and titanate could be effectively controlled by various parameters of the hydrothermal method. Recently, some groups have reported the fabrication of titania and titanate layers on titanium substrates for biomedical applications. ${ }^{32}$ However, reports on surface topography affecting the interaction between bone and the implant and further deciding the preloading integration success rate of implants are still rare.

Earlier studies have shown that, when artificial materials were implanted into bone defects, they were generally encapsulated by the fibrous tissue, leading to their isolation from the surrounding bone, and did not integrate with the bone. This has been a critical problem in their applications to bone repair. In order to overcome this problem, bioactive materials that can spontaneously bond to the living bone without the formation of surrounding fibrous tissue have been studied widely. Bioactivity of the implant materials means that a layer of apatite, which usually consists of nanosized crystals of carbonate-ion-containing apatite, is induced to form on the surface of the implant materials. Due to its similarity in composition with the mineral phase in bone, this apatite layer can be recognized by bone tissue, and therefore promotes integration with bone. ${ }^{33-36}$ Previous studies have shown that simulated body fluid (SBF) has ion concentrations nearly equal to that of the human blood plasma and can well reproduce the in vivo apatite formation. ${ }^{36,37}$ Thus, the most efficient method for investigating the biological behavior of the biomaterials and the biomimetic deposition of the apatite layer is to immerse the samples into SBF. For bone implant, it is very important to find out the relationship between the surface characteristics of materials and the bioactivity of materials as well as the synergistic effect between different surface characteristics in enhancing apatite-forming ability. In this work, we developed a hydrothermal method to tune the morphologies of titanate layers on titanium surfaces. Their surface morphology and chemical composition were characterized using several techniques used in materials science. To show that the surface structures prepared by the hydrothermal method are suitable for use as bone implants, the effect of the morphologies of titanate layers on their ability to promote apatite formation was investigated. In vitro cell culture was also performed to demonstrate the materials' biocompatibility and to explore the biomedical applications of the prepared biomaterials.

\section{Materials and methods Preparation of samples}

Commercially pure titanium foils $(99.7 \%$, Aldrich) with the dimension of $10 \mathrm{~mm} \times 10 \mathrm{~mm} \times 2 \mathrm{~mm}$ were employed as substrates. They were immersed in an etchant containing $10 \%(\mathrm{v} / \mathrm{v}) \mathrm{HNO}_{3}, 1 \%(\mathrm{v} / \mathrm{v}) \mathrm{HF}$, and deionized (DI) water for 1 minute. Finally, the Ti samples were cleaned consecutively with acetone, ethyl alcohol, and DI water, each for 15 minutes under ultrasonication. The $\mathrm{Ti}$ foils were then placed in a Teflon-lined stainless steel vessel containing $10 \mathrm{M} \mathrm{NaOH}$ solution, the vessel was sealed and hydrothermally treated at $100^{\circ} \mathrm{C}$ and $140^{\circ} \mathrm{C}$ for 2 hours and at $150^{\circ} \mathrm{C}$ for 3 hours, and cooled naturally in air. To obtain a fixed film, the treated Ti substrates were initially rinsed with $5 \mathrm{M} \mathrm{NaOH}$, and the $\mathrm{NaOH}$ solution was gradually diluted with DI water to $\mathrm{pH} 7$. The obtained samples were directly dried in air, and are referred to as Ti100, Ti140, and Ti150, respectively.

\section{Immersion test in SBF}

SBF was used as an incubation solution for apatite formation on the prepared coatings. The ion concentrations of SBF were nearly equal to that of human blood plasma. SBF was prepared according to Kokubo's formulation by dissolving reagent-grade mixtures of $\mathrm{NaCl}, \mathrm{NaHCO}_{3}, \mathrm{KCl}$, $\mathrm{K}_{2} \mathrm{HPO}_{4} \cdot 3 \mathrm{H}_{2} \mathrm{O}, \mathrm{MgCl}_{2} \cdot 6 \mathrm{H}_{2} \mathrm{O}, \mathrm{CaCl}_{2}$, and $\mathrm{Na}_{2} \mathrm{SO}_{4}$ in DI water and buffering at $\mathrm{pH} 7.40$ with tris-hydroxymethyl aminomethane and $1.0 \mathrm{M}$ hydrochloric acid $(\mathrm{HCl})$ at $36.5^{\circ} \mathrm{C} .{ }^{36} \mathrm{All}$ the prepared samples were soaked in SBF at a constant temperature of $36.5^{\circ} \mathrm{C}$. As controls, untreated Ti plates were also soaked in SBF under the same conditions. All the samples were kept in the vertical position inside polypropylene tubes (one per tube). After soaking in SBF for different periods, ie, 7 days and 14 days, the samples were gently washed with DI water and dried at room temperature.

\section{Characterization}

The surface morphologies of all the samples were observed by scanning electron microscopy (SEM, Hitachi-S4800, Hitachi Ltd., Tokyo,, Japan) at $20 \mathrm{kV}$, and elemental analysis 
was carried out by an X-ray energy dispersion spectroscopy (EDS) attachment that was directly connected to the SEM. The corresponding crystal structural characterization of all the samples was done using their X-ray diffraction (XRD, PANalytical X'pert PRO, PANalytical Ltd., Almelo, the Netherlands) patterns with a $\mathrm{Cu} \mathrm{K} \alpha$ radiation source at $40 \mathrm{kV}$ and $30 \mathrm{~mA}$. The XRD patterns were collected over the $2 \theta$ range $20^{\circ}-60^{\circ}$ using a step size of 0.0167 and a time per step of 20. Fourier transform infrared spectroscopy (FTIR, Nicolet-740) was employed in transmission mode using the $\mathrm{KBr}$ technique to analyze the chemical composition of the coatings before and after soaking in SBF. The Raman measurements at room temperature were performed using a Raman spectrometer (Renishaw, UV-vis Raman System 1000) equipped with an $\operatorname{Ar}^{+}$laser $(\lambda=514.5 \mathrm{~nm})$. The structure of the formed nanotubes was characterized using transmission electron microscopy (TEM, Philips-FEI, Tecnai F30, the Netherlands). The nanotubes were scraped off from the Ti substrate, dispersed in ethanol, and deposited on a copper grid for TEM analysis. The water contact angles were measured at ambient temperature using a contact angle instrument (OCA 40, Data Physics, Corporation, Filderstadt, Germany) with a drop volume of $5 \mu \mathrm{L}$. DI water (Millipore, $18 \mathrm{M} \Omega \cdot \mathrm{cm}$ ) was employed as the medium for the contact angle measurements. All reported contact angles are the average of measurements on four drops at different locations of the sample surface.

\section{In vitro cell culture}

Preosteoblast cells MC3T3-E1 were expanded in alpha minimum essential medium (Thermo Fisher Scientific, Waltham, MA, USA) containing 10\% fetal bovine serum (Life Technologies, Carlsbad, CA, USA) and 1\% penicillin/ streptomycin (Life Technologies, Carlsbad, CA, USA). Cells were incubated at $37^{\circ} \mathrm{C}$ in a humidified atmosphere with $5 \%$ $\mathrm{CO}_{2}$, and the medium was replaced every 48 hours. After culturing in the growth medium till $80 \%-90 \%$ confluence, they were harvested to seed onto the coated samples.

Sterilized samples $(10 \mathrm{~mm} \times 10 \mathrm{~mm} \times 2 \mathrm{~mm})$ were transferred into 24-well plastic culture plates. MC3T3-E1 cells were seeded onto the prepared samples at a density of $1 \times 10^{5}$ cells per sample. Cells were incubated in the culture medium ( $1 \mathrm{~mL} /$ well) at $37^{\circ} \mathrm{C}$ under $5 \% \mathrm{CO}_{2}$ atmosphere for 1 day, 3 days, and 7 days, with the medium replaced every 3 days until being harvested.

Cell viability and proliferation were measured by 3-(4,5-dimethylthiazol-2-yl)-2,5-diphenyl tetrazolium bromide (MTT; Sigma) staining. At desired time points, the cell-seeded samples were incubated in MTT solution ( $5 \mathrm{mg} / \mathrm{mL}$ MTT in cell culture medium) in a $5 \% \mathrm{CO}_{2}$ incubator at $37^{\circ} \mathrm{C}$ for 4 hours. The intense purple formazan derivative formed via cell metabolism was eluted and dissolved in dimethylsulfoxide (DMSO) with the dosage of $400 \mu \mathrm{L}$ per well. The absorbance was measured at $570 \mathrm{~nm}$ with a reference wavelength of $690 \mathrm{~nm}$ using a microplate reader (BioTek Synergy4). The cell number was correlated to the optical density.

Fluorescein diacetate (FDA) molecular probe was used as a viable cell marker to investigate the growth of cells on the prepared coatings. After 3 days of culture, the cell-seeded samples were washed with PBS, incubated with $30 \mu \mathrm{g} / \mathrm{mL}$ FDA solution for 15 minutes, and washed with PBS. The positive cells were observed immediately under a laser confocal microscope (Olympus FluoView 1000).

\section{Results and discussion Micromorphology and compositions of the Ti foil after hydrothermal treatment}

Typical SEM micrographs of the Ti plates before and after hydrothermal treatment at $100^{\circ} \mathrm{C}-150^{\circ} \mathrm{C}$ are shown in Figure 1A-D. After hydrothermal treatment for 2 hours at $100^{\circ} \mathrm{C}$, a porous network structure with the pore size of 200-300 $\mathrm{nm}$ in diameter was observed on the surface of Ti100 (Figure 1B). Figure 1C shows that, once the temperature was increased to $140^{\circ} \mathrm{C}$, bumped crystals sheets were formed on the surface of Ti140. After hydrothermal reaction at $150^{\circ} \mathrm{C}$ for 3 hours (Ti150), a porous structure consisting of tiny fiber-like crystals with an average diameter approximately $20 \mathrm{~nm}$ was obtained (Figure 1D). The TEM image in Figure 1E shows that the fiber-like crystals have typical nanotubular structure, which are made of multilayered sheets. From the above observation, it indicates that the surface microtopography of Ti substrate can be controlled effectively by varying the hydrothermal reaction temperature and time. The EDS spectrum shown in Figure 1F identifies that the elemental composition of prepared samples under the different hydrothermal conditions consists mainly of oxygen, titanium, and sodium.

The crystalline structures of the prepared coatings were identified by XRD, as shown in Figure 2. The characteristic diffraction peaks of the samples at $24^{\circ}, 28^{\circ}$, and $48^{\circ}$ are assigned to the crystal of sodium titanate $\left(\mathrm{Na}_{2} \mathrm{Ti}_{3} \mathrm{O}_{7}\right),{ }^{26}$ as shown by the curves $b-d$ in Figure 2. The corresponding peaks in curves $\mathrm{b}$ and $\mathrm{c}$ are extremely inconspicuous because the sodium titanate layers on Ti100 and Ti140 are too thin to be detected by XRD. The intensities of these characteristic 

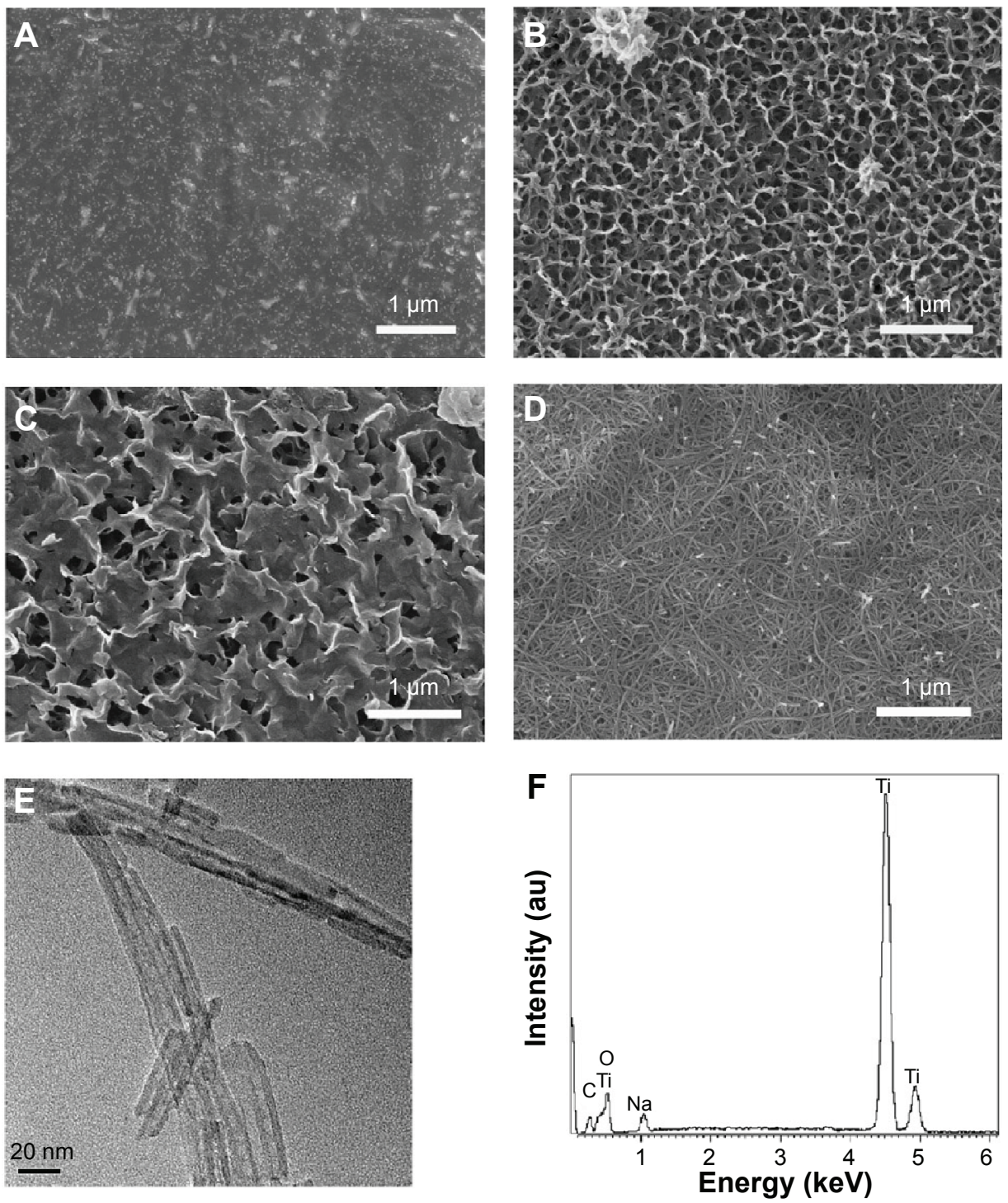

Figure I SEM micrographs, TEM images and EDS spectrum of Ti plates with hydrothermal treatment at different temperatures.

Notes: SEM micrographs (A) Untreated Ti. (B) Til00. (C) Til40. (D) Til50. (E) TEM images of the nanotubes obtained from hydrothermal treatment at I50 ${ }^{\circ} \mathrm{C}$ for 3 hours (Til50). (F) EDS spectrum of the hydrothermally treated samples.

Abbreviations: SEM, scanning electron microscopy; TEM, transmission electron microscopy; EDS, energy dispersion spectroscopy.

peaks in curve $d$ increase clearly because of the increased thickness of this sodium titanate layer by raising the reaction temperature and time. The above change is also confirmed by the Raman spectra in Figure 3. With increasing reaction temperature and time, the Raman spectra of hydrothermally treated samples show more obvious bands near $165 \mathrm{~cm}^{-1}$, $278 \mathrm{~cm}^{-1}, 445 \mathrm{~cm}^{-1}, 696 \mathrm{~cm}^{-1}$, and $900 \mathrm{~cm}^{-1}$, which are characteristic of $\mathrm{Na}_{2} \mathrm{Ti}_{3} \mathrm{O}_{7}{ }^{38}$

\section{Wettability of the prepared samples}

Images of water droplets lying on flat prepared samples surfaces and the corresponding contact angle values are shown in Figure 4. The water contact angle for the untreated $\mathrm{Ti}$ plates is approximately $58.4^{\circ} \pm 1 \cdot 1^{\circ}$. After the hydrothermal treatment, the water droplet quickly spread and wetted all the treated samples (Ti100, Ti140, and Ti150), and the water contact angles decreased to near $0^{\circ}$, indicating that all the treated samples were superhydrophilic.

\section{Biological performance of prepared samples}

\section{Bone-like apatite-forming behavior}

To evaluate the bioactivity of the samples prepared by hydrothermal treatment, the formation behavior of the apatite layer on the prepared samples was examined by immersing them in SBF for up to 14 days. After incubation in SBF for 7 days 


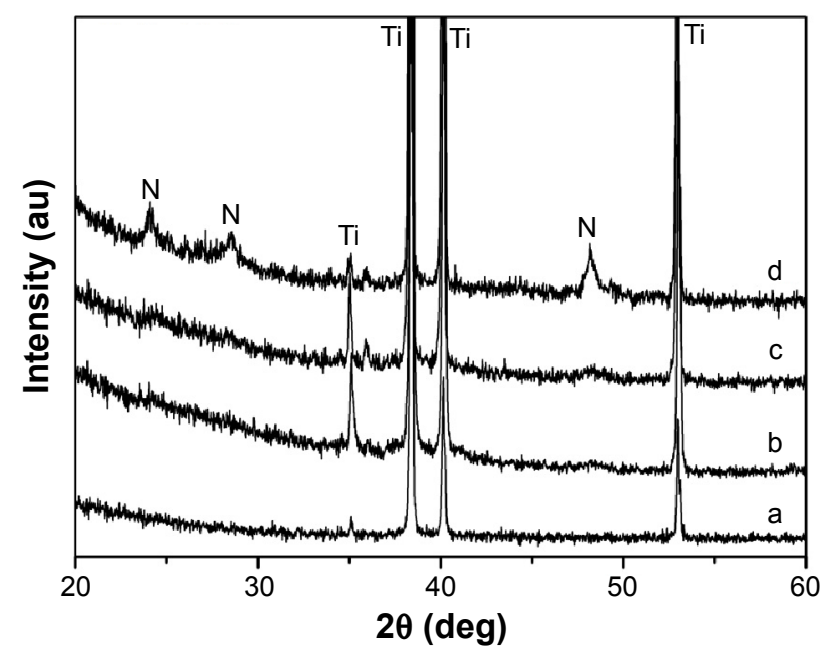

Figure $2 \times R D$ spectra of samples with hydrothermal treatment at different temperatures.

Notes: (a) Untreated Ti. (b) Til00. (c) Til40. (d) Til 50.

Abbreviations: $\mathrm{N}, \mathrm{Na}_{2} \mathrm{Ti}_{3} \mathrm{O}_{7}$; XRD, X-ray diffraction.

and 14 days, the surface morphology of the samples with or without hydrothermal treatment was observed by SEM, as shown in Figure 5. Among all the samples, there were no new layers formed on the surfaces of Ti100, Ti140, Ti150, and untreated Ti foil after soaking in SBF for 7 days. When the immersion time was increased to 14 days, the entire surface of the Ti150 sample was covered by newly formed layers (Figure 6D). The newly formed layers exhibited a network structure consisting of nanosheets. Unfortunately, there was still no apatite layer formation on the surface of Ti100, Ti140, and untreated Ti for the same soaking time (Figure 6A-C). The elemental composition of the newly formed layers consisted mainly of $\mathrm{Ca}, \mathrm{P}, \mathrm{Ti}, \mathrm{O}$, and a small

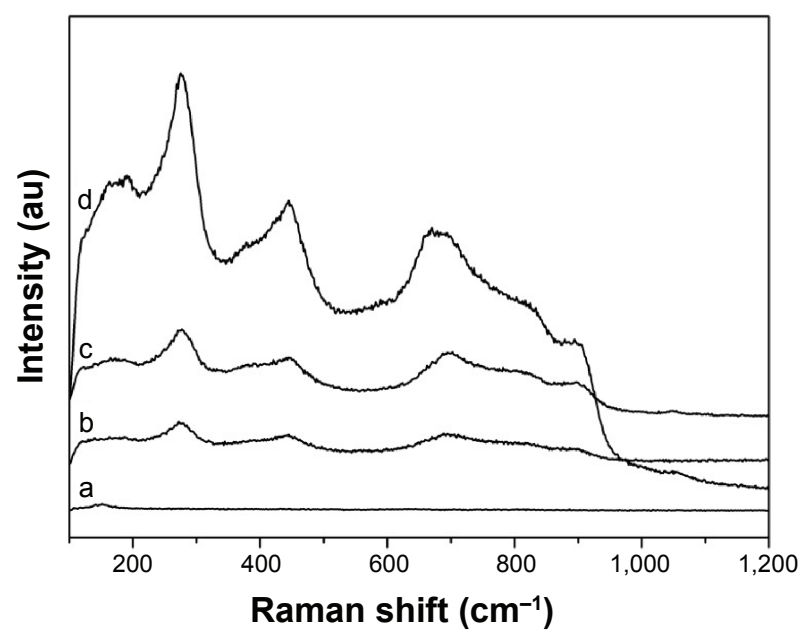

Figure 3 Raman spectra of the samples with hydrothermal treatment at different temperatures.

Notes: (a) Untreated Ti. (b) Til00. (c) Til40. (d) Til50.

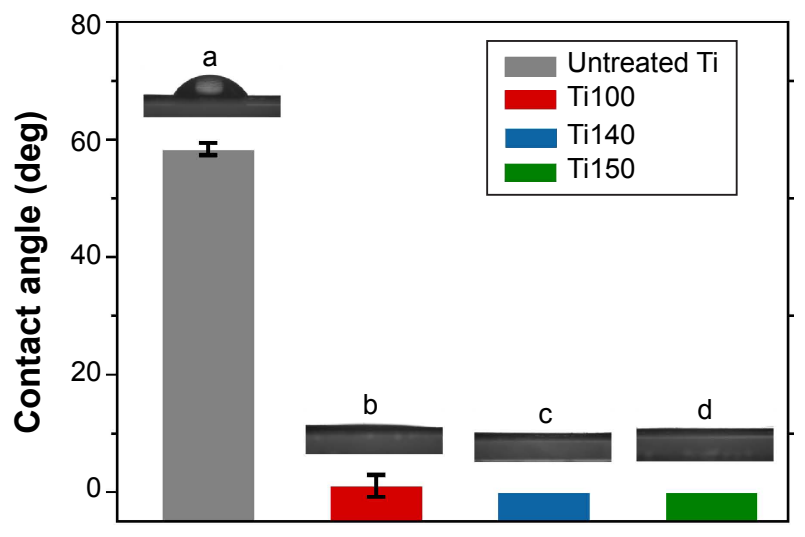

Figure 4 Water contact angles of the samples with hydrothermal treatment at different temperatures.

Notes: (a) Untreated Ti. (b) Til00. (c) Til40. (d) Til50.

fraction of magnesium, and the average $\mathrm{Ca} / \mathrm{P}$ molar ratio was approximately 1.41 , as evaluated from the EDS spectra of Figure 7A. The FTIR spectrum of the layer newly formed on the samples surface is shown in Figure 7B. The peak at $1,035 \mathrm{~cm}^{-1}$ is assigned to the $\mathrm{P}-\mathrm{O}$ asymmetric stretching mode (v3) of the $\mathrm{PO}_{4}{ }^{3-}$ group in apatite. ${ }^{39}$ The bending modes of the phosphate $\mathrm{O}-\mathrm{P}-\mathrm{O}$ bonds are detected at $604 \mathrm{~cm}^{-1}$ and $565 \mathrm{~cm}^{-1} .{ }^{40}$ The peaks corresponding to the $\mathrm{CO}_{3}{ }^{2-}$ vibrational mode are found at $874 \mathrm{~cm}^{-1}, 1,421 \mathrm{~cm}^{-1}$, and $1,460 \mathrm{~cm}^{-1}$, which means that the $\mathrm{PO}_{4}{ }^{3-}$ sites of the apatite structure are partially substituted by $\mathrm{CO}_{3}{ }^{2-}$ groups (B-type substitution). ${ }^{37}$ Based on the EDS and FTIR results, it can be concluded that newly formed layer is calcium-deficient carbonated apatite with traces of magnesium, which has similar mineral composition as that of natural bones.

Furthermore, apatite formation can be identified from the Raman spectra since the intensity of the characteristic peak at $960 \mathrm{~cm}^{-1}$ originating from the $v 1$ symmetric stretching of the $\mathrm{P}-\mathrm{O}$ mode is appreciable. ${ }^{39}$ It failed to show the formation of the apatite layers on the surfaces of all samples after the immersion in SBF for 7 days (Figure 8A). After soaking in SBF for 14 days, the Raman peak at $960 \mathrm{~cm}^{-1}$, which is assigned to the apatite phase, suggests that the deposited layer on the surface of Ti150 possesses a crystalline structure (Figure $8 \mathrm{~B}$ ), which can be verified by the SEM images in Figure 6D. It is noteworthy that only the hydrothermally treated sample with a nanotubular structure could induce apatite deposition with continued soaking in SBF for 14 days.

\section{Cell proliferation and morphology of the prepared coatings}

MTT assay was used to compare the cell viability of the prepared samples (Figure 9). In order to investigate the 

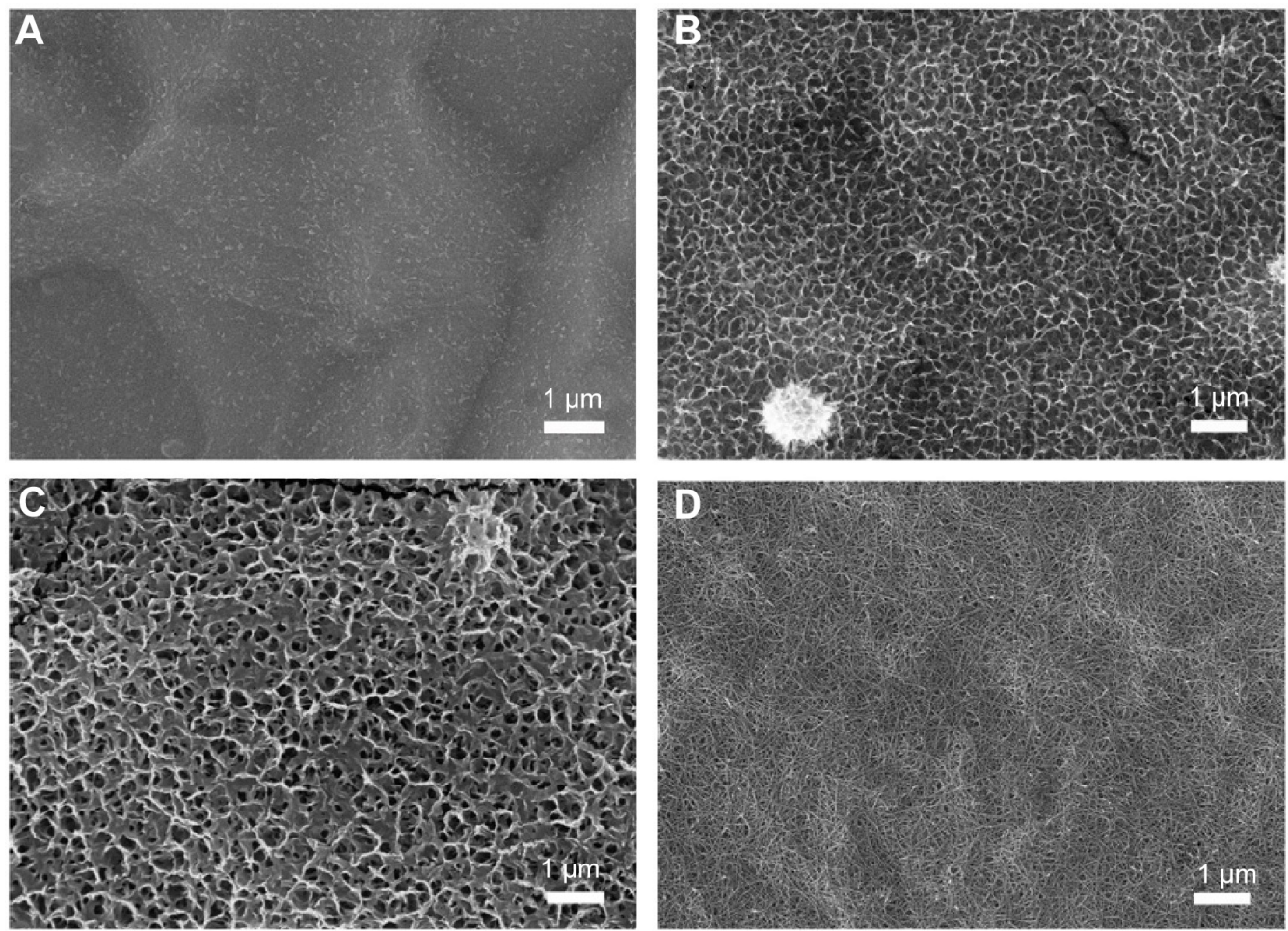

Figure 5 SEM micrographs of the various samples after soaking in SBF for 7 days. Notes: (A) Untreated Ti. (B) Til00. (C) Til40. (D) Til50.

Abbreviations: SEM, scanning electron microscopy; SBF, simulated body fluid.
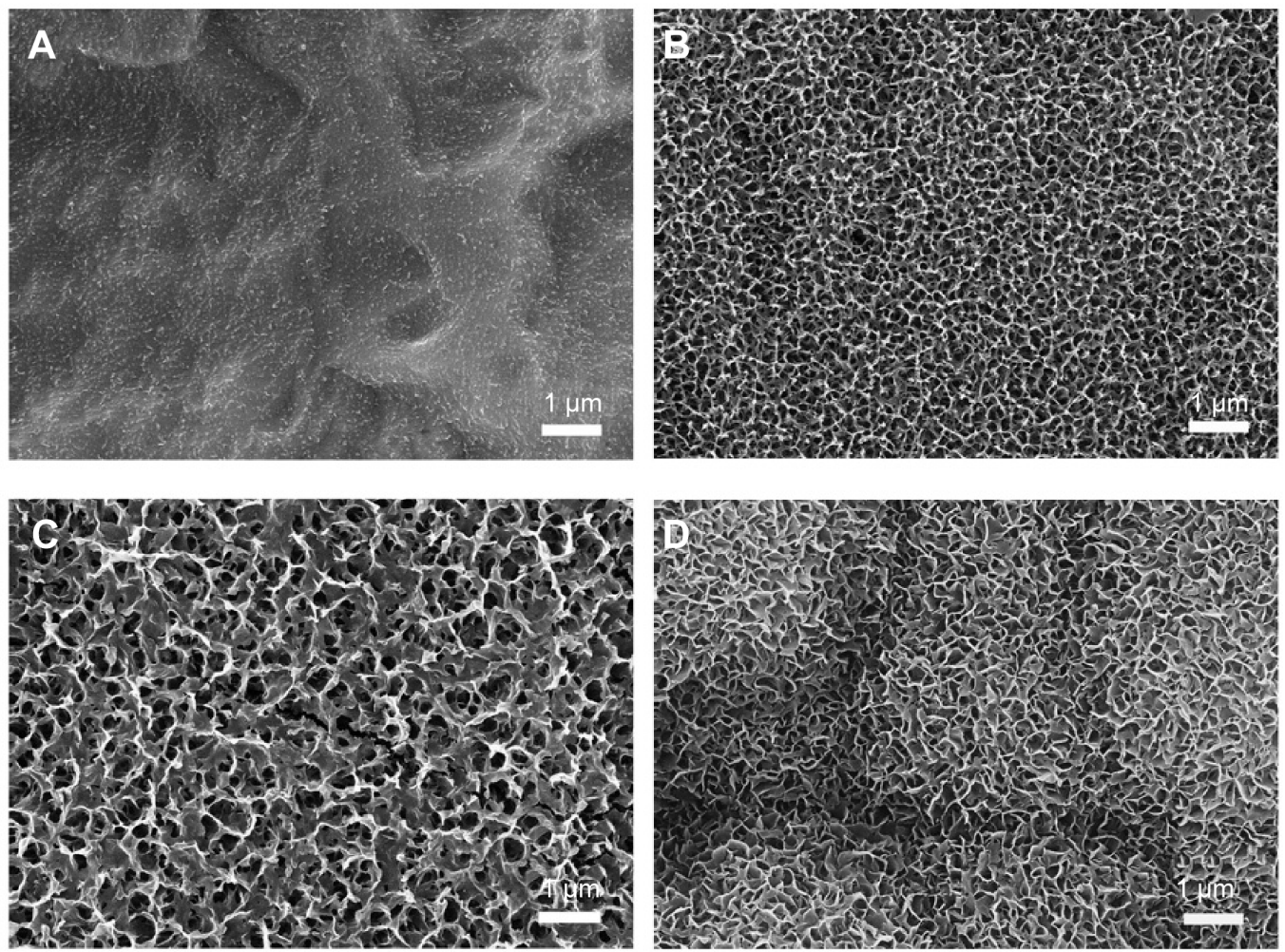

Figure 6 SEM micrographs of the various samples after soaking in SBF for 14 days. Notes: (A) Untreated Ti. (B) Til00. (C) Til40. (D) Til50.

Abbreviations: SEM, scanning electron microscopy; SBF, simulated body fluid. 

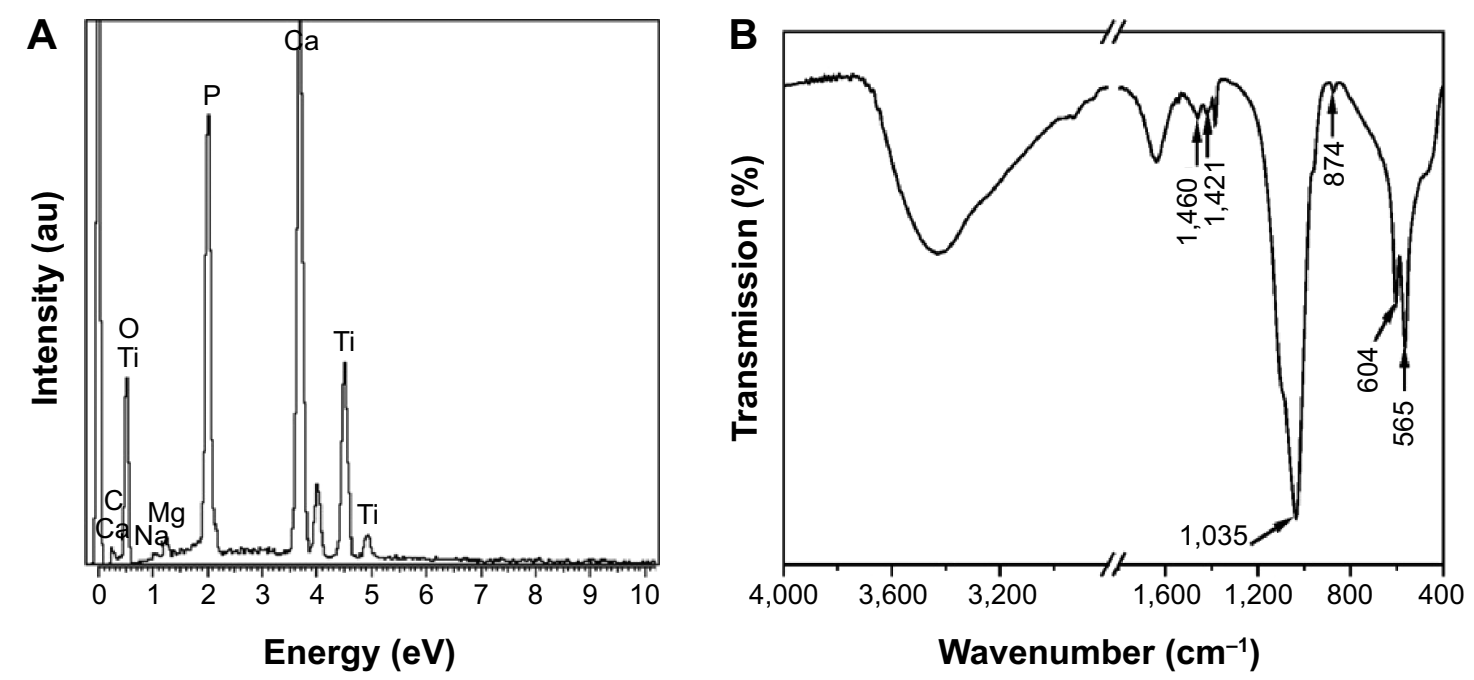

Figure 7 EDS spectrum (A) and FTIR spectrum (B) of the newly formed layer on the Til50 after soaking for 14 days. Abbreviations: EDS, energy dispersion spectroscopy; FTIR, Fourier transform infrared spectroscopy.

cell response to the surface characteristics of the prepared samples, the adhesion and proliferation of preosteoblast MC3T3 cells were measured. There was a clear temporal proliferation profile after 1 day, 3 days, and 7 days of culture on all the samples. As shown in Figure 9, the viability of preosteoblast MC3T3 cells cultured on the Ti100, Ti140, and Ti150 samples appeared significantly higher than that of the untreated Ti at 7-day time points $(P<0.05)$. In addition, sample Ti150 showed the highest proliferation at 7-day time points, showing a viability $\sim 1.4$ times higher than in the untreated Ti. These results clearly indicate that Ti150 with a nanotubular structure may promote cell proliferation, allowing more cells to grow in a congenial environment.

Laser confocal microscopy was used to study the dynamics of cell adhesion and spreading on the prepared samples after fluorescent straining with FDA, as shown in Figure 10 MC3T3 cells on untreated Ti show an uneven morphology after 3 days in culture. Compared to the cells adhering to untreated Ti, MC3T3 cells adhered to and spread on the treated samples more uniformly. As shown in Figure 10, a larger number of MC3T3 cells got attached to and fully covered the surface of sample Ti150.

In general, the biological performance of biomaterials is attributed to their surface structure and composition. Bonelike apatite formation on the surface of biomaterials is the essential requirement for an artificial material to integrate with the surrounding bonetissue. ${ }^{35,36}$ Some previous studies have suggested the catalytic effect of $\mathrm{Ti}-\mathrm{OH}$ groups for apatite nucleation, and these functional groups are abundant on the surface of sodium titanate or titania. ${ }^{16,41}$ In our
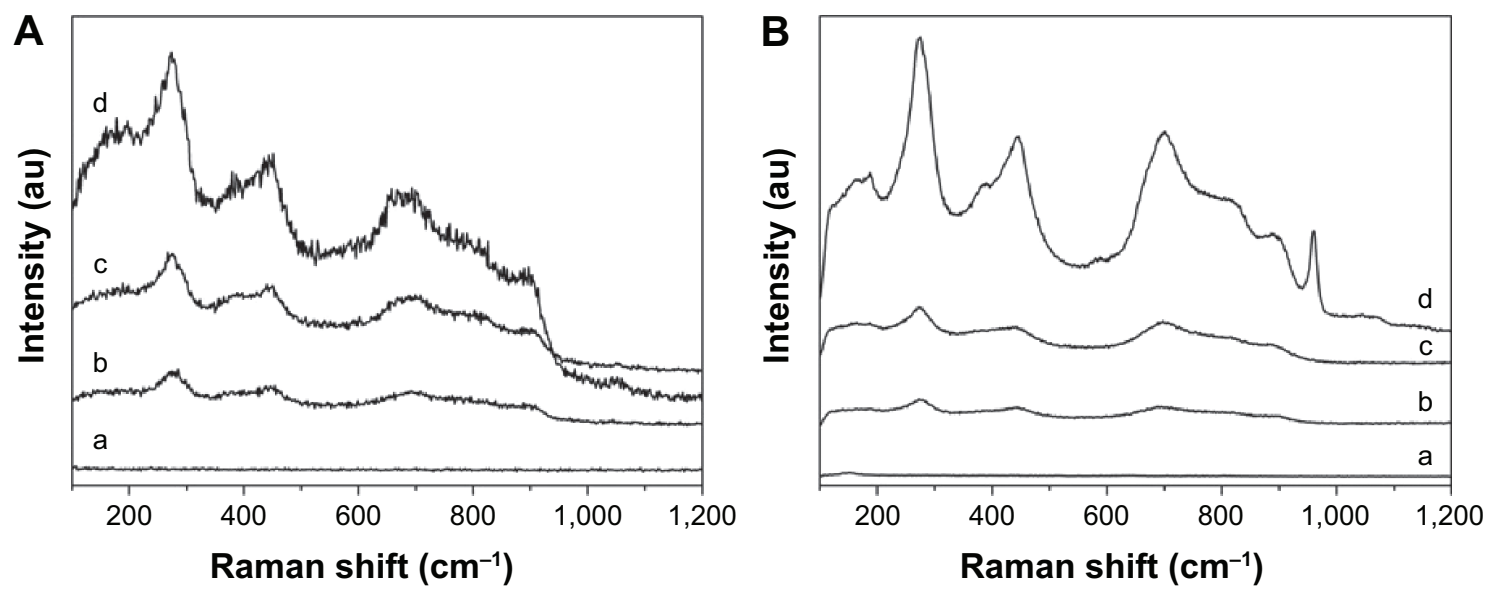

Figure 8 Raman spectra of the various samples after soaking in SBF for (A) 7 days and (B) 14 days.

Notes: (a) Untreated Ti. (b) Til00. (c) Til40. (d) Til50.

Abbreviation: SBF, simulated body fluid. 


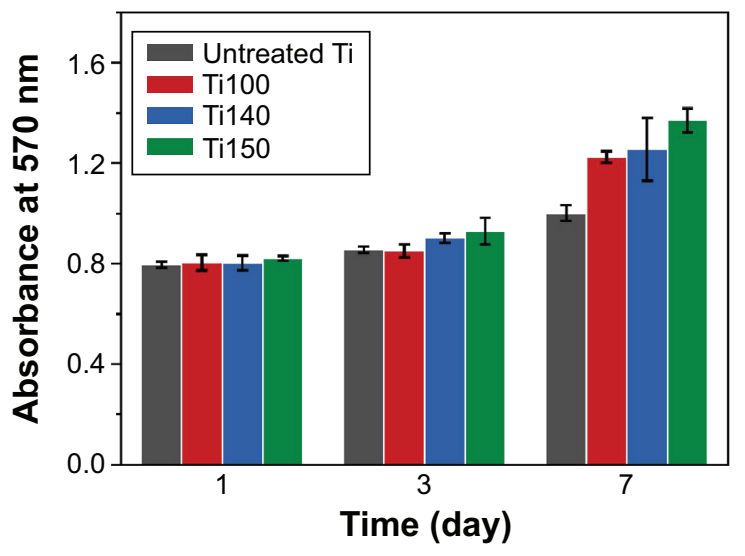

Figure 9 Preosteoblast MC3T3 cell proliferation on untreated Ti, Til00, Til 40, and Til 50 for I day, 3 days, and 7 days. experiments, the XRD and Raman results (Figures 2 and 3) show that sodium titanate $\left(\mathrm{Na}_{2} \mathrm{Ti}_{3} \mathrm{O}_{7}\right)$ layers are formed on the Ti substrate after hydrothermal treatment in a $10 \mathrm{M} \mathrm{NaOH}$ solution. In other words, all the treated samples had the same surface composition. Interestingly, only the hydrothermally treated samples with a unique nanotubular structure (Ti150) could induce apatite formation after being soaked in SBF for 14 days, while no apatite layers were observed on the surfaces of the samples without the nanotubular structure (untreated Ti, Ti100, and Ti140) after immersion for the same time. It indicates that the formed nanotubular structure could improve the apatite-forming ability effectively. Our results show that no nanotubular structure is obtained after
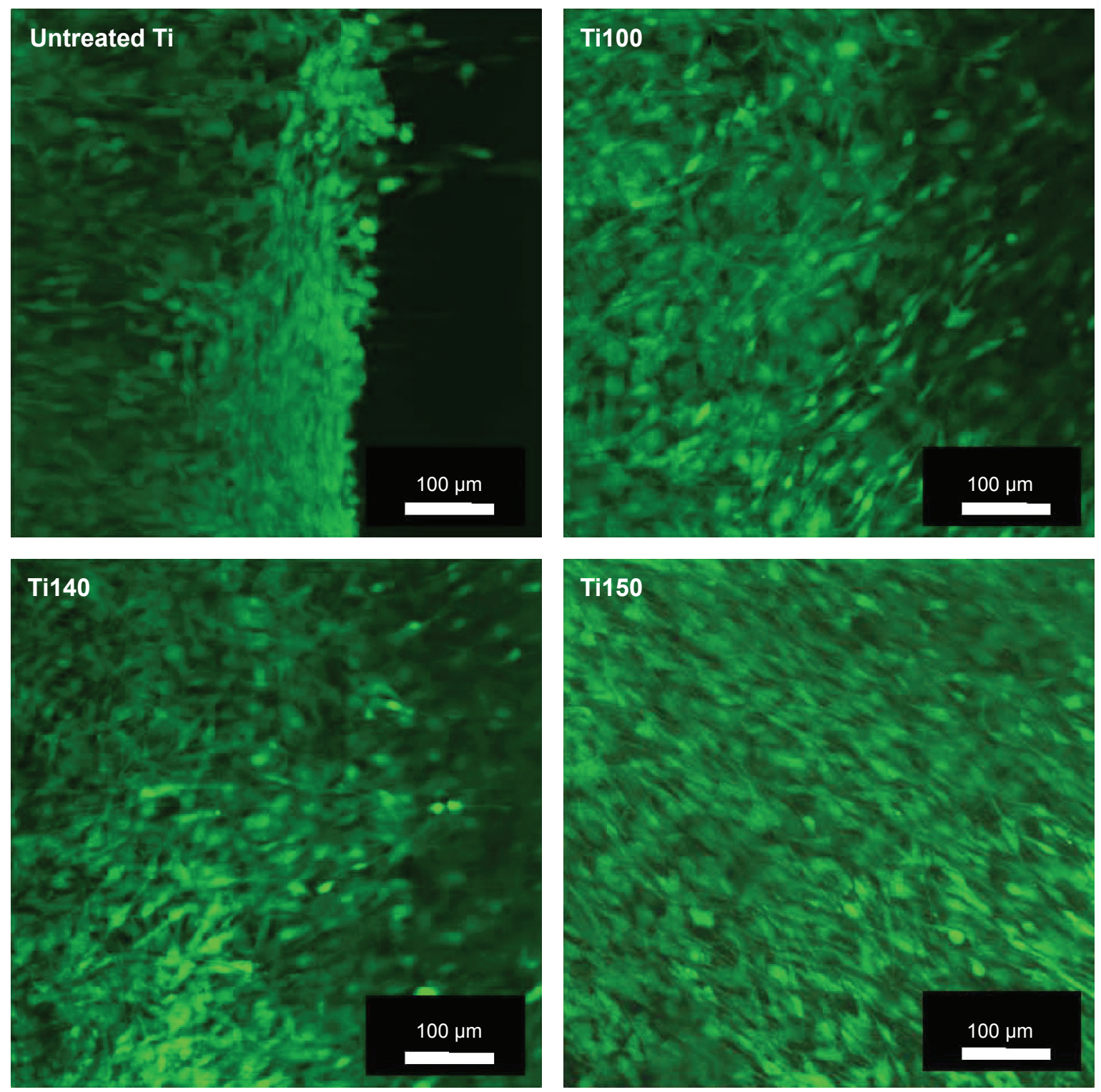

Figure 10 Laser confocal micrographs of preosteoblast MC3T3 cells incubated on untreated Ti, Til00, Til40, and Til50 stained with FDA molecular probe for 3 days. Abbreviation: FDA, fluorescein diacetate. 
hydrothermal treatment at $100^{\circ} \mathrm{C}$ and $140^{\circ} \mathrm{C}$; however, such structures are observed at $150^{\circ} \mathrm{C}$ (Figure 1). It indicates that temperature can accelerate the formation of the nanotubular structure. These observations show that temperature is the most important factor that affects the surface microtopography of the Ti plate during hydrothermal treatment. It can be concluded from these findings that the surface microtopography of the materials, which has a significant impact on the formation of the apatite, can be well controlled by the reaction temperature in hydrothermal treatment. Obviously, the samples Ti150 with the nanotubular structure possess a large effective surface area. Meanwhile, when the treated samples are soaked in $\mathrm{SBF}, \mathrm{Na}^{+}$ions released from the sodium titanate layer exchange with $\mathrm{H}_{3} \mathrm{O}^{+}$ions to form Ti-OH groups. It can be inferred that the large surface area of Ti150 induces more $\mathrm{Ti}-\mathrm{OH}$ groups to form on the Ti surface. The large number of $\mathrm{Ti}-\mathrm{OH}$ groups formed immediately reacts with the $\mathrm{Ca}^{2+}$ ions in SBF to accelerate the formation of amorphous calcium titanate. This calcium titanate later combines with the phosphate ions in SBF to form amorphous calcium phosphate. Once the apatite nuclei are formed, they can grow spontaneously by consuming the surrounding calcium and phosphate ions due to the high supersaturation in SBF, and finally transform into bone-like apatite with a small concentration of $\mathrm{Mg}$ and $\mathrm{Na}$, which is the most stable phase in the body fluid, as shown in Figure 6D.

All experiments of cell culture have demonstrated that the treated sample could improve MC3T3 cell adhesion, spreading, and proliferation. It is noted that after hydrothermal treatment, although superhydrophilic titanate surfaces are formed on all the treated samples (Ti100, Ti140, and Ti150), Ti150 with the nanotubular surface structure showed better cell response, indicating excellent biocompatibility of the nanotubular structure. When the nanotubular structure is formed, the surface area of Ti150 increases distinctly. It could provide sufficient space for cell anchoring, spreading, and proliferation. In the meantime, it has been proposed that cell filopodia can recognize the nanoscale topography. ${ }^{14}$ The nanotubular structure formed on Ti150 presents a very high affinity to the cells incubated on it.

Based on the results of SBF immersion test and cell culture test in this study, it is clear that the nanotubular structure plays a very important role in enhancing the apatite-forming ability and cell response. These findings clearly demonstrate that the prepared Ti150 samples have excellent biocompatibility and high bioactivity, which are attributed to the special nanoscale structure and a suitable surface composition.
Conclusion

Bioactive materials have been known to spontaneously integrate with living bones by promptly forming bone-like apatite on their surfaces, which is important for fast bone regeneration. In this study, chemical treatment with high concentrations of an alkaline solution at high temperature was employed to rapidly modify the surface morphology and composition of titanium materials to enhance their biological performance. The samples with different surface characteristics were soaked in SBF solution and seeded by preosteoblast MC3T3 cells for various times to investigate their biological performance. Experimental results indicated that, compared with the traditional smooth, untreated Ti implants, titanate materials with the nanotubular structure had a great promoting effect on the apatite-forming ability and improved preosteoblast MC3T3 cell response due to their special nanoscale structure and suitable surface composition. This relatively simple methodology and the outstanding performance of the nanostructured titanate film would be convenient for future bone and hard-tissue engineering applications.

\section{Acknowledgments}

The authors are grateful for the support from the National Natural Science Foundation of China under Grants 51203108, 51073113, 91027039, 51373110 and 21321062, the National Scientific Support Program of China under Grants 2012BAI07B09, the Natural Science Foundation of Jiangsu Province of China under Grants BK2011355 and BK20130313, and the Natural Science Foundation of the Jiangsu Higher Education Institutions of China under Grants 11KJB430011 and 10KJA540046.

The authors also acknowledge support from the Priority Academic Program Development of Jiangsu Higher Education Institutions (PAPD), Qing Lan Project for Excellent Scientific and Technological Innovation Team of Jiangsu Province (2012), and Project for Jiangsu Scientific and Technological Innovation Team (2013).

\section{Disclosure}

The authors report no conflicts of interest in this work.

\section{References}

1. Brunette DM, Tengvall P, Textor M, and Thomsen P. Titanium in Medicine. NY, Berlin Heidelberg: Spinger-Verlag; 2001.

2. Long M, Rack HJ. Titanium alloys in total joint replacement-a materials science perspective. Biomaterials. 1998;19(18):1621-1639.

3. Khan MA, Williams RL, Williams DF. In-vitro corrosion and wear of titanium alloys in the biological environment. Biomaterials. 1996;17(22): $2117-2126$ 
4. Liu XY, Paul KC, Ding CX. Surface modification of titanium, titanium alloys, and related materials for biomedical applications. Mater Sci Eng R Rep. 2004;47(3-4):49-121.

5. Zhang L, Chen Y, Rodriguez J, Fenniri H, Webster TJ. Biomimetic helical rosette nanotubes and nanocrystalline hydroxyapatite coatings on titanium for improving orthopedic implants. Int J Nanomedicine. 2008; 3(3):323-333.

6. Dong W, Zhang T, Epstein J, et al. Multifunctional Nanowire Bioscaffolds on Titanium. Chem Mater. 2007;19(18):4454-4459.

7. Yang W, Xi X, Si Y, Huang S, Wang J, Cai K. Surface engineering of titanium alloy substrates with multilayered biomimetic hierarchical films to regulate the growth behaviors of osteoblasts. Acta Biomater. 2014; 10(10):4525-4536.

8. Tanaka SI, Fukushima Y, Nakamura I, Tanaki T, Jerkiewicz G. Preparation and characterization of microporous layers on titanium by anodization in sulfuric acid with and without hydrogen charging. ACS Appl Mater Interfaces. 2013;5(8):3340-3347.

9. Wang H, Lin C, Hu R, Zhang F, Lin L. A novel nano-micro structured octacalcium phosphate/protein composite coating on titanium by using an electrochemically induced deposition. J Biomed Mater Res A. 2008; 87A(3):698-705.

10. Schuler M, Trentin D, Textor M, Tosatti SG. Biomedical interfaces: titanium surface technology for implants and cell carriers. Nanomedicine. 2006;1(4):449-463.

11. Puleo DA, Nanci A. Understanding and controlling the bone-implant interface. Biomaterials. 1999;20(23-24):2311-2321.

12. Ito $\mathrm{Y}$, Kajihara M, Imanishi Y. Materials for enhancing cell adhesion by immobilization of cell-adhesive peptide. J Biomed Mater Res. 1991; 25(11):1325-1337.

13. Yang L, Sheldon BW, Webster TJ. Orthopedic nano diamond coatings: Control of surface properties and their impact on osteoblast adhesion and proliferation. J Biomed Mater Res A. 2003;91A(2):548-556.

14. Xing H, Komasa S, Taguchi Y, Sekino T, Okazaki J. Osteogenic activity of titanium surfaces with nanonetwork structures. Int J Nanomedicine. 2014;9:1741-1755.

15. Rani VVD, Vinoth-Kumar L, Anitha VC, Manzoor K, Deepthy M, Shantikumar VN. Osteointegration of titanium implant is sensitive to specific nanostructure morphology. Acta Biomater. 2012;8(5):1976-1989.

16. Chen CY, Ozasa K, Katsumata K, Maeda M, Okada K, Matsushita N. Bioactive titanium oxide-based nanostructures prepared by one-step hydrothermal anodization. J Phys Chem C. 2012;116(14): 8054-8062.

17. Xin Y, Jiang J, Huo K, Hu T, Chu PK. Bioactive $\mathrm{SrTiO}_{3}$ nanotube arrays: strontium delivery platform on Ti-based osteoporotic bone implants. ACS Nano. 2009;3(10):3228-3234.

18. Lv L, Liu Y, Zhang $\mathrm{P}$, et al. The nanoscale geometry of $\mathrm{TiO}_{2}$ nanotubes influences the osteogenic differentiation of human adiposederived stem cells by modulating H3K4 trimethylation. Biomaterials. 2015;39:193-205.

19. Jamting AK, Bell M, Swain MV, Wielunski LS, Clissold R. Measurement of the micro mechanical properties of sol-gel $\mathrm{TiO}_{2}$ films. Thin Solid Films. 1998;332(1-2):189-194.

20. Costacurta S, Maso GD, Gallo R, Guglielmi M, Brusatin G, Falcaro P. Influence of temperature on the hotocatalytic activity of sol-gel $\mathrm{TiO}_{2}$ films. ACS Appl Mater Interfaces. 2010;2(5):1294-1298.

21. Uhm SH, Song DH, Kwon JS, Im SY, Han JG, Kim KN. Timedependent growth of $\mathrm{TiO}_{2}$ nanotubes from a magnetron sputtered $\mathrm{Ti}$ thin film. Thin Solid Films. 2013;547(1):181-187.

International Journal of Nanomedicine

\section{Publish your work in this journal}

The International Journal of Nanomedicine is an international, peerreviewed journal focusing on the application of nanotechnology in diagnostics, therapeutics, and drug delivery systems throughout the biomedical field. This journal is indexed on PubMed Central, MedLine, CAS, SciSearch $®$, Current Contents ${ }^{\circledR} /$ Clinical Medicine,
22. Ohtsua N, Itoa A, Saitoa K, Hanawab T. Characterization of calcium titanate thin films deposited on titanium with reactive sputtering and pulsed laser depositions. Surf Coat Technol. 2007;201(18):7686-7691.

23. Chen HW, Huanga KC, Hsua CY, et al. Electrophoretic deposition of $\mathrm{TiO}_{2}$ film on titanium foil for a flexible dye-sensitized solar cell. Electrochim Acta. 2011;56(23):7991-7998.

24. Yui T, Mori Y, Tsuchino T, et al. Electrophoretic deposition of unstable colloidal suspensions for superhydrophobic surfaces. Langmuir. 2011; 27(7):4156-4163.

25. Kin BH, Lee JY, Choa YH, Higuchi M, Mizutani N. Preparation of $\mathrm{TiO}_{2}$ thin film by liquid sprayed mist CVD method. Mater Sci Eng B. 2004;107(3):289-294.

26. Li S, Zhang G, Guo D, Yu L, Zhang W. Anodization fabrication of highly ordered $\mathrm{TiO}_{2}$ nanotubes. J Phys Chem C. 2009;113(29):12759-12765.

27. Lai Y, Lin C, Wang H, Huang J, Zhuang H, Sun L. Superhydrophilicsuperhydrophobic micropattern on $\mathrm{TiO}_{2}$ nanotube films by photocatalytic lithography. Electrochem Commun. 2008;10(3):387-391.

28. Guo YP, Lee NH, Oh HJ. Structure-tunable synthesis of titanate nanotube thin films via a simple hydrothermal process. Nanotechnology. 2007; 18(29):295608.

29. Wu D, Liu J, Zhao XN, Li AD, Chen YF, Ming NB. Sequence of events for the formation of titanate nanotubes, nanofibers, nanowires, and nanobelts. Chem Mater. 2006;18(2):547-553.

30. Tsai CC, Teng H. Regulation of the physical characteristics of titania nanotube aggregates synthesized from hydrothermal treatment. Chem Mater. 2004;16(22):4352-4358.

31. Lai Y, Tang Y, Huang J, et al. Multi-functional hybrid protonated titanate nanobelts with tunable wettability. Soft Matter. 2011;7:6313-6319.

32. Lin L, Wang H, Ni M, et al. Enhanced osteointegration of medical titanium implant with surface modifications in micro/nanoscale structures. J Orthop Transl. 2014;2:35-42.

33. Ohtsuki C, Kushitani H, Kokubo T, Kotani S, Yamamuro T. Apatite formation on the surface of ceravital-type glass-ceramic in the body. J Biomed Mater Res. 1991;25(11):1363-1370.

34. Kokubo T. Bioactive glass ceramics: properties and applications. Biomaterials. 1991;12(2):155-163.

35. Kokubo T, Kim HM, Kawashita M. Novel bioactive materials with different mechanical properties. Biomaterials. 2003;24(13):2161-2175.

36. Kokubo T, Takadama H. How useful is SBF in predicting in vivo bone bioactivity. Biomaterials. 2006;27(15):2907-2915.

37. Zadpoor AA. Relationship between in vitro apatite-forming ability measured using simulated body fluid and in vivo bioactivity of biomaterials. Mater Sci Eng C. 2014;35:134-143.

38. Qamar M, Yoon CR, Oh HJ, et al. Effect of post treatments on the structure and thermal stability of titanate nanotubes. Nanotechnology. 2006;17(24):5922-5929.

39. Chen DY, Jordan EH, Gell M, Wei M. Apatite formation on alkalinetreated dense $\mathrm{TiO}_{2}$ coatings deposited using the solution precursor plasma spray process. Acta Biomater. 2008;4(3):553-559.

40. Koutsopoulos S. Synthesis and characterization of hydroxyapatite crystals: a review study on the analytical methods. J Biomed Mater Res. 2002;62(4):600-612.

41. Uchida M, Kim HM, Kokubo T, Nakamura T. Apatite-forming ability of titania gels with different structures. J Biomed Mater Res. 2003;64A(1): 164-170.

Journal Citation Reports/Science Edition, EMBase, Scopus and the Elsevier Bibliographic databases. The manuscript management system is completely online and includes a very quick and fair peer-review system, which is all easy to use. Visit http://www.dovepress.com/ testimonials.php to read real quotes from published authors. 